\title{
Advantages of using indocyanine green in liver transplantation: a narrative review
}

\author{
Bo Dai $^{1 \#}$, Nida El Islem Guissi ${ }^{2 \#}$, Lydia Frenzel Sulyok ${ }^{3,4}$, Mitchell G. Bryski ${ }^{3,4}$, Yiqing Wang ${ }^{2}$, \\ Dongjin Wang ${ }^{1}$, Sunil Singhal ${ }^{3,4}$, Huiming Cai $^{2,5}$ \\ ${ }^{1}$ Department of Cardio-Thoracic Surgery, Nanjing Drum Tower Hospital, the Affiliated Hospital of Nanjing University Medical School, Nanjing, \\ China; ${ }^{2}$ Department of Biomedical Engineering, College of Engineering and Applied Sciences, Nanjing University, Nanjing, China; ${ }^{3}$ Center for \\ Precision Surgery, Perelman School of Medicine at the University of Pennsylvania, Philadelphia, PA, USA; ${ }^{4}$ Department of Surgery, Perelman \\ School of Medicine at the University of Pennsylvania, Philadelphia, PA, USA; ${ }^{5}$ Nanjing Nuoyuan Medical Devices Co., Ltd., Nanjing, China \\ Contributions: (I) Conception and design: H Cai, S Singhal; (II) Administrative support: D Wang; (III) Provision of study materials or patients: D \\ Wang; (IV) Collection and assembly of data: B Dai; (V) Data analysis and interpretation: B Dai, NEI Guissi; (VI) Manuscript writing: All authors; (VII) \\ Final approval of manuscript: All authors. \\ \#These authors contributed equally to this work. \\ Correspondence to: Huiming Cai. Fuqian South Road 18, Nanjing 211514, China. Email: chm9166@aliyun.com; Sunil Singhal. University of \\ Pennsylvania, Philadelphia, PA 19104, USA. Email: sunil.singhal@uphs.upenn.edu. Dongjin Wang. Zhongshan Road 321, Nanjing 210000, China. \\ Email: gldjw@163.com.
}

Objective: To assess the role of indocyanine green in liver transplantation and to lay the foundation for its application in clinical practice.

Background: Liver transplantation offers the best prognosis for patients with end-stage liver disease. However, this invasive procedure involves multiple well-known challenges, including complications due to graft rejection and dysfunction, surgical risks, and critical postoperative management. Intraoperative methods to assess graft function rely on conventional methods, such as blood chemistries and Doppler ultrasound. However, these methods are limited in their abilities to assess liver conditions, predict functional outcomes of the graft, and prevent surgical complications. Thus, identifying a more effective and comprehensive detection method is necessary.

Methods: The information used to write this narrative review was collected from the references' opinions and conclusions.

Conclusions: Indocyanine green can effectively monitor blood flow during surgery, evaluate donor graft function, and monitor the recipients functional status during and after surgery. It may also help surgeons to predict the prognosis of patients throughout the liver transplantation process, from assessing patients for liver transplantation status to postoperative management. Therefore indocyanine green should be routinely used in liver transplantation to help re-organize the transplant waiting list and improve the surgical outcomes of liver transplantation patients.

Keywords: Indocyanine green (ICG); liver transplantation (LT); liver allocation; transplant assessment; surgical complications

Submitted Nov 12, 2021. Accepted for publication Jan 12, 2022.

doi: 10.21037/atm-21-6650

View this article at: https://dx.doi.org/10.21037/atm-21-6650 


\section{Introduction}

The high mortality rate of end-stage liver diseases is a global health issue. In the United States, approximately 30,000 patients die from chronic liver disease each year, and the incidence of such deaths has been increasing. Liver transplantation (LT) is the only curative treatment for end-stage liver disease (1); however, the results are disappointing. The mortality rate at 30 days postoperatively is as high as $3 \%$, which increases to approximately $7 \%$ at 90-day postoperatively (2,3). LT complications include dysfunctional grafts, unpredictable surgical complications, and delayed postoperative intervention (4-6). This review discusses indocyanine green (ICG) perfusion and related tests as complementary methods to assess graft function in real time, before, during, and after transplantation, in order to predict surgical complications.

Graft function is most commonly evaluated by the ChildPugh score and the Model for End-Stage Liver Disease (MELD) score. However, two parameters in the ChildPugh system, ascites and encephalopathy, are subjective. Additionally, the international normalized ratio (INR) in the two systems cannot sufficiently reflect coagulopathy and consequently liver function, and meanwhile ignores interlaboratory variation $(7,8)$. Blood tests for liver-specific enzymes, like the ratio between concentrations of alanine aminotransferase and aminotransaminase (ALT/AST), does not express liver function accurately enough to predict surgical outcomes. Imaging methods, such as computed tomography (CT), can accurately calculate the liver volume, but cannot reflect the function of liver cells. Furthermore, CT imaging does not evaluate hepatocellular failure caused by biliary obstruction (9).

ICG is a safe, low-cost, and commonly used injectable dye that fluoresces in the near-infrared region. When excited by near-infrared light, ICG emits fluorescence that can be detected in real-time during operations. Typically, after it is intravenously injected to the patient, ICG binds to serum proteins and is rapidly uptaken by hepatic cells and tumor tissue. This NIR fluorescent probe accumulates in tumors, via the enhanced permeability and retention (EPR) effect (10), hence achieving a better tumor detection. Upon irradiation with NIR light, ICG absorbs the light at $800 \mathrm{~nm}$, emitting a strong fluorescence at a wavelength of $830 \mathrm{~nm}$, thus improving tumor localization. Once the fluorescence is collected by the relevant instrument, it can be displayed on the screen of the instrument after being processed by the computer for rapid image registration, so as to provide accurate surgical guidance. The molecular formula of ICG has been described by Vos et al. (11). Compared with visible light, near-infrared light penetrates more effectively through living tissue. ICG was approved by the Food and Drug Administration (FDA) for clinical use and has played a critical role in several applications, including assessing perfusion in colon anastomoses and breast reconstruction $(12,13)$, detecting sentinel lymph nodes $(14,15)$, and intraoperative localization of tumors (16). It has also been shown to successfully predict liver reserve function prior to liver resection (17). As such, ICG could be used in conjunction with traditional liver function tests. Intraoperatively, ICG offers clear visualization and assessment of liver function. Moreover, the use optical molecular imaging can help doctors assess the approximate surgical location. Combined with RGDICG molecular probes, it can quickly locate the surgical area. Moreover, RGD-ICG molecular probes can help doctors reduce exploration time, improve exploration efficiency, and thereby reduce operation time. These advantages in assessing liver function before and during liver resection and transplantation have led some transplant centers to commonly adopt ICG perfusion during LT. Interestingly, ICG perfusion of donor grafts can reasonably assess graft function, perform real-time intraoperative navigation, and even predict postoperative risk. In this review paper, we reviewed the data on ICG in LT. As shown in Table 1, using ICG has been shown to be an effective and convenient method to assess liver function compared with other methods. Based on the data, we recommend ICG as a safe, cost-effective tool that provides valuable clinical data in LT to help improve patient prognosis in end-stage liver disease. Previous literature on ICG was mainly limited to: (I) liver function reserve test (II) vascular patency and (III) tumor identification. However, to the best of our knowledge, systematic reports describing ICG's unique advantages specifically in liver transplantation are not available. These advantages are as follows: (I) ICG can make up for the insufficiency of MELD score and allow a more reasonable allocation of organs. (II) It helps evaluate the liver status and (III) reduce the rate of useless liver transplantation. The patient's prognosis (IV) can help predict early postoperative complications and provide early warning regarding the medical treatment. We present the following article in accordance with the Narrative Review reporting checklist (available at https://atm.amegroups. com/article/view/10.21037/atm-21-6650/rc). 
Table 1 Method usually used in evaluation of liver function

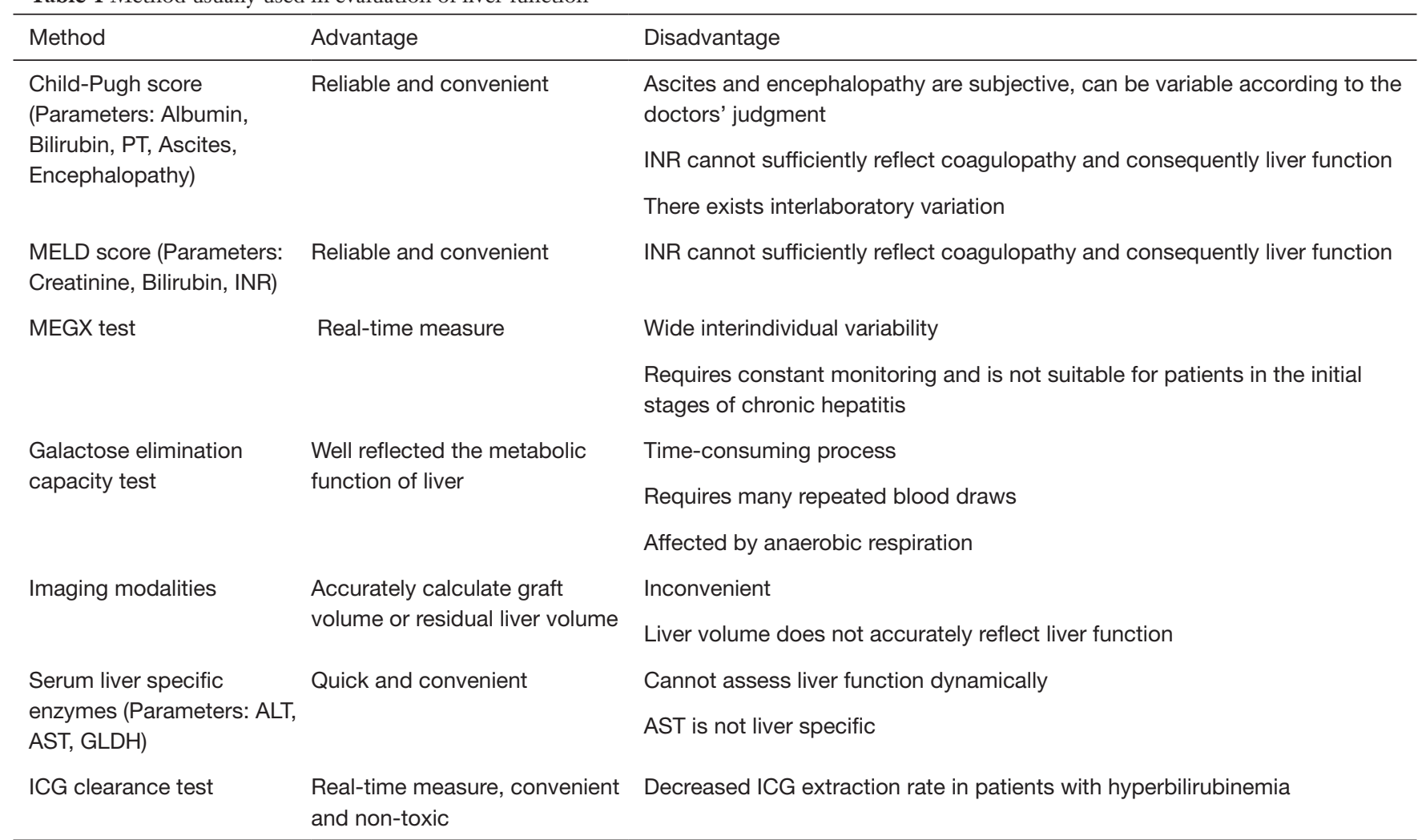

INR, international normalized ratio; PT, Prothrombin activity; MEGX, monoethylglycinexylidide; ALT, alanine aminotransferase; AST, aspartate aminotransferase; GLDH, glutamate dehydrogenase; MELD, model for end stage liver disease; ICG, indocyanine green.

Table 2 Sources used for this overview

PubMed search: 1966-January 2001. Key words: Indocyanine green (ICG); liver transplantation; liver allocation; transplant assessment; surgical complications. Review of the Literature; Authorship; Meta-analysis; Narrative overview

Discuss with experts in the ICG field

Information collection and surgical observation of liver transplantation patients

Author's experience of ICG in liver surgery

The author's own experience in writing ICG related research papers and reviews

ICG, indocyanine green.

\section{Methods}

We searched the PubMed database from 1966 to January 2021 using the following keywords: indocyanine green, liver transplantation, liver allocation, transplant assessment, and surgical complications. The information used to write this paper was collected from the sources listed in Table 2.

\section{Discussion}

\section{Pre-transplantation benefits of ICG}

Assessing the status of LT candidates by ICG clearance test

The primary limitation of LT is the shortage of donor livers. 
Long wait times significantly reduce patient survival before and after transplantation (18). Depending on the condition of the recipient, proper organ allocation is important to improve the overall transplantation survival rate. The MELD score accurately predicts the short-term survival and mortality risk in patients with end-stage liver disease and is widely used for liver distribution worldwide (19), but it is ineffective in $15-20 \%$ of patients (20). Ecochard et al. added metabolic liver function tests to improve the prediction accuracy of the MELD score; in a study of 560 cirrhotic patients listed for transplantation, the ICG clearance rate was evaluated. This improved the prognostic and survival evaluation with high sensitivity and specificity, indicating that an ICG clearance test can be used as a pretransplant receptor test program to assist MELD scoring, hence more effectively distribute organs (21). For patients with acute liver failure (ALF), the plasma disappearance rate of indocyanine green (ICG-PDR) exhibited a close relationship with the patient's survival rate, which suggests that ICG can help surgeons determine whether patients are good candidates for LT (22). Moreover, ICG-PDR is a safe and non-invasive test that can be conveniently performed at the patient's bedside.

\section{Evaluating the graft's function by ICG retention rate}

Due to recent advances in surgery and improved perioperative care, LT offers many patients the best prognosis, making this surgery widely accepted worldwide and further increasing the demand for donor livers $(23,24)$. In order to meet the demand, many transplantation centers have expanded the criteria for transplant donation, including the use of marginal donor livers with steatosis, chronic hepatitis, and even tumors for critical cases (25). Assessing the function of these potential grafts is the first step in LT. Although each transplantation center appropriately prioritizes this analysis of donor liver function, there still exists a lack of accurate, quantitative methods.

Tang et al. performed ICG clearance testing on 90 brain-dead donors before liver procurement, including 45 cases of livers with varying degrees of cirrhosis, acute hepatic necrosis, hepatocellular carcinoma and/ or hepatitis B virus (HBV)-associated cirrhosis. The results showed that the ICG retention rate at 15 minutes (ICGR 15 ) $>11.0 \%$ had significantly lower 3 -month graft survival rates (26). Zarrinpar et al. measured ICGPDR in 53 donors, including 11 with poor-quality livers, and observed that ICG-PDR was the only effective donor predictor of 7-day graft survival (27). Wang et al. investigated the 15 -minute detection rate of ICG (ICGR15D) in 106 liver donors, including 22 cases of mild fatty liver and 20 cases of moderate to severe fatty liver. The study found that the ICGR15D increased according to the severity of donor fatty liver, and those with $>20 \%$ of fatty degeneration (ICGR15D $4.5 \pm 1.1$ ) had a higher risk of transplantation failure and postoperative complications (28). Therefore, ICG can be used as a pretransplant graft function evaluation factor to predict the prognosis of transplant patients. Globally, ICG clearance represent the most common test used to perioperatively evaluate liver function in case of liver resection surgery and liver transplantation (29). To determine ICG clearance, the standard method used is the ex vivo photometric analysis of several arterial blood samples obtained in a short time frame $(15 \mathrm{~min})$ following the intravenous injection of ICG. ICGPDR and ICGR15 are the two parameters usually used to evaluate ICG clearance. For donors with long ICG clearance time, pre-transplant treatment, such as antiviral therapy, can be considered, which may effectively preempt transplant failure.

\section{Intraoperative function of ICG}

\section{Preventing biliary complications through ICG fluorescence cholangiography}

Biliary complications are the Achilles heel of LT, with incidence rates ranging from $23-43.8 \%$ in different transplantation centers. The two most common complications are bile leaks (8.5-46\%) and strictures (14.7$37.5 \%)$, with resulting mortality rates of $1-3 \%(30-32)$. Abnormal bile duct anatomy around the hilum and postoperative inflammatory reactions associated with biliary complications, make these challenges more severe. Fluorescence imaging technology provides the ability to visualize biliary structures through the liver hilar fat and surrounding tissues. Furthermore, it makes it easier to identify the cystic duct without a tedious dissection of Calot's triangle (33). Since 2008, this approach has been increasingly adopted (34).

Mizuno et al. conducted a study of 108 donors and recipients undergoing living-donor liver transplantation (LDLT), with a mean follow-up time of 58 months. In the control group (without ICG imaging guidance), six patients had postoperative bile leaks (5.6\%), 15 had biliary strictures (13.9\%), and 3 three donors had bile leaks $(2.7 \%)$. In the ICG fluorescence cholangiography 
group, there were no biliary complications in the donors or recipients (35). It was observed that ICG biliary fluorescence imaging made it easier for surgeons to identify the demarcation of the biliary tree in the donor, thereby preventing biliary complications (36). Hong et al. reported the use of ICG near-infrared fluorescence cholangiography to assist during a pediatric LDLT case. They found the optimal bile duct division point by realtime ICG fluorescence cholangiography and reported no biliary complications following surgery (37). Nonetheless, optimizing cholangiography may require the development of individualized standards based on the patient's metabolic status, which is a direction that requires further research.

\section{Preventing vascular complications by using ICG with ultrasound}

LT-related vascular complications usually include hemorrhage, stenosis, thrombosis, and anastomoses. Although rare, these complications have a mortality rate of $13 \%$ in LDLT (13\%) and a mortality rate of $7 \%$ in various series of deceased donor liver transplantation (DDLT) (4,38-40). Without timely intervention arterial thrombosis alone results in a mortality rate of greater than 50\% (41). Insufficient anastomoses and stenosis are highly associated with these complications. Although intraoperative Doppler ultrasound (IDUS) is used to ensure effective vascular reconstruction in LDLT, it cannot visualize the anastomosis in real time (42). Kubota et al. used ICG for imaging of reconstructed vessels in three patients who had undergone LDLT. They demonstrated that, in addition to monitoring bile production in the transplanted liver graft, ICG can help the surgeon clearly visualize the reconstructed hepatic artery and portal vein (42). ICG fluorescence has been shown to be a valuable adjunct to ultrasound to guarantee the patency of reconstructed vessels.

\section{Observing reperfusion of graft}

Primary graft dysfunction (PGD) is a clinical situation that comprises initial poor function (IPF) and primary nonfunction (PNF), and when accompanied by reperfusion injury, PGD results in irreversible graft failure $(43,44)$. Traditionally, there has been no effective method to assess and predict graft dysfunction intraoperatively after graft implantation. More recently, ICG imaging has been used to evaluate liver parenchymal perfusion. Figueroa et al. used ICG fluorescence imaging to analyze graft perfusion. They assessed perfusion in 72 patients during LT and found that an abnormal intraoperative ICG fluorescence pattern (non-homogeneous fluorescence) accompanied the high occurrence of PGD after LT (45). LT hypoperfusion caused by portal vein thrombosis can be detected by ICG imaging. After performing ICG fluorescence imaging to confirm graft perfusion, Kawaguchi et al. observed insufficient perfusion on the segmental surface, which was ultimately diagnosed as portal vein thrombosis (46). Hepatic venous outflow obstruction of the graft can lead to a reduction in portal uptake and sinusoidal perfusion, resulting in necrosis or insufficient regeneration of related venoocclusive regions (VOR), and ultimately leading to graft dysfunction $(47,48)$. In order to prevent postoperative graft dysfunction caused by VOR, major hepatic vein tributaries sometimes need to be reconstructed (49). Although preoperative three-dimensional computed tomography (CT) can estimate the relative regional liver volumes with postoperative VOR, it cannot alert the surgeon in real time if the patient requires venous reconstruction. Hashimoto et al. evaluated the decrease of sinusoidal perfusion in VOR in the remnant liver after LT by detecting ICG using nearinfrared spectroscopy. There is a notable difference in ICG detection between VOR and non-VOR, but there also exists patient-to-patient differences (50). On this basis, Kawaguchi et al. directly used the fluorescence imaging function of ICG to observe the surface of the graft. The imaging clearly identifies VOR from non-VOR, and provides ICG intake values which can effectively help doctors decide whether they need to reconstruct hepatic vein tributaries (51).

\section{Monitoring portal venous flow by ICG elimination rate} Insufficient graft size is a major issue that is often accompanied by poor surgical outcomes and patient death; however, has been shown to be acceptable given adequate portal blood pressure (PVP) $(52,53)$. This situation can be effectively alleviated by regulating portal venous flow (PVF). However, some conditions such as cirrhosis with collaterals of the portal vein, would prevent PVP from correctly reflecting the optimal PVF. Therefore, it is necessary to establish a reliable indicator to predict the optimal PVF during LDLT. Hori et al. used the ICG elimination rate (kICG) to confirm that optimal PVF can be achieved during LDLT. They observed significant improvements in the LT outcomes when the final PVP $<15 \mathrm{mmHg}$ and $\mathrm{kICG}>3.1175 \times 10^{-4} \mathrm{~g}$. The kICG/graftweight value is a reliable intraoperative predictor and indicator, according to which surgeons can adjust the PVF via surgery or other means to ensure the safety of the transplantation (54). 


\section{Postoperative function of ICG}

\section{Predicting early mortality and complications by ICG clearance rate}

Graft dysfunction is the primary indication for early re-transplantation, and LT mortality is usually caused by vascular thrombosis, sepsis, and acute rejection, among other complications. Moreover, the recurrence of hepatocellular carcinoma (HCC) after LT, is rather common with a rate of about $10-15 \%$ (55). Several risk factors play a role in HCC recurrence and patient's survival. For instance, the more the size and number of tumors exceeds Milan criteria, the greater the risk. Microvascular invasion is closely related to HCC recurrence and reduces the survival of patients. In addition, poor tumor differentiation is also a related risk factor for postoperative recurrence. However, recipient's tumor marker alphafetoprotein (AFP) prior to transplantation is negatively correlated with the prognosis of LT (56). Hepatic artery thrombosis (HAT) alone results in a re-transplantation rate of up to $75 \%$ (57) and mortality rate of around 50\% (41). Thus, predicting early graft dysfunction can provide treating physicians with necessary information to promptly adjust postoperative treatments, in order to improve the survival rate of patients. Du et al. retrospectively analyzed 178 patients who underwent LDLT, and their results showed that a minimum ICG clearance rate constant K (m-KICG) $<0.100 /$ minute was the strongest predictor of early graft loss after postoperative day 3 (sensitivity, $100 \%$; specificity, $97.2 \%$ ) (58). Olmedilla et al. conducted a prospective analysis between the ICG-PDR and early graft function after LT, and showed that the survival rate was significantly lower when the 1-hour plasma disappearance value was below $10.8 \% /$ minute (59). Klinzing et al. analyzed ICG-PDR within 6 hours after Intensive Care Unit (ICU) admission and calculated the MELD score before LT; they found that when MELD $>25$ and ICG-PDR $<20 \% /$ minute, the ICU stay time and hospital mortality were significantly longer. They suggested that ICG-PDR can help predict the outcome and risk stratification after LT and create an optimal intensive care management strategy to avoid early postoperative complications (such as acute rejection, sepsis etc.) (60). Levesque et al. measured ICG-PDR in $72 \mathrm{LT}$ recipients in the first 5 days after LT and found that a ICGPDR cutoff level of approximately $12.85 \%$ /minute predicts serious complications. The sequential changes of ICGPDR indicated early severe complications with low values of around $8.8 \% \pm 4.5 \% /$ minute, and acute rejection activity with a progressive reduction value from $25.5 \% \pm 4.8 \%$ to $10.3 \% \pm 2.5 \%$ /minute $(61)$.

As described before, HAT is the most severe complication after LT. Doppler ultrasonography, which has a high sensitivity (approximately 92\%), is the first choice to investigate HAT. Levesque et al. used ICG-PDR in conjunction with ultrasonography, and observed that a low value of ICG-PDR with abnormal ultrasound images was usually an indicator of HAT occurrence. Additionally, they found that with a normal value of ICG-PDR, angiography was unnecessary in emergency situations, suggesting that ICG-PDR may be more sensitive than angiography (62). Sun et al. also investigated ICG-PDR in 115 LT patients, and found that hepatic arterial complications and rates of pneumonia were higher in the ICG-PDR $<18 \% /$ minute group compared to a control (63). Collectively, these studies show that the ICG clearance rate can provide a reliable, convenient, and low-cost postoperative test to predict mortality and surgical complications. According to the ICG clearance rate results, doctors can detect, prevent, or alleviate complications. ICG-PDR may be a good additional tool for physicians to use in their treatment plan.

\section{Detecting graft function and regeneration by ICG clearance tests}

Measuring the early function of grafts after LT can help strengthen clinical management and further improve transplant outcomes. Some laboratory indicators such as the aspartate aminotransferase (AST), alanine aminotransferase (ALT), $\mathrm{pH}$, and prothrombin time (PT) can reflect the function of the graft to a certain extent, but they are not representative. ICG clearance tests can be used as complementary tests to predict graft functionality by evaluating functional hepatocyte levels and effective hepatic blood flow. Vos et al. reported that ICG-PDR can accurately predict early graft function (64). Hori et al. assessed the reliability of KICG (ICG elimination rate constant) value up to 28 days after LDLT. In the first 24 hours after LT, there were already significant differences between recipients with or without good graft function, reflecting different effective hepatic blood flow rates, which is crucial for liver regeneration (65). Liver regeneration after LDLT closely affects the transplant response in both donors and recipients. Jochum et al. used functional tests such as galactose elimination capacity (GEC), lidocaine half-life and ICG half-life as biomarkers to evaluate liver regeneration in the first 3 months after LT in both donors and recipients. The results showed that ICG and GEC 
differed significantly between donors (ICG, -9.1\%; GEC, $+59.3 \%$ ) and recipients (ICG, $-63.7 \%$; GEC, $+16.3 \%$ ), and the lidocaine half-life showed no significant changes (66), which suggests that ICG can be reliably used to test postoperative liver regeneration.

\section{Evaluating optimal systemic hemodynamics by ICG kinetics}

Systemic hemodynamic changes cause long-term portal hypertension in patients with advanced cirrhosis. Even after transplantation, this situation is difficult to alleviate (67). PVF is affected by systemic hemodynamics, and excessive PVP can cause LT failure. Timely and accurate assessment of systemic hemodynamics to regulate PVF is important for the success of transplantation.

\section{Conclusions}

To assess liver function and prevent surgical complications, ICG offers many advantages in LT compared to other available approaches. It is effective at low doses, metabolized rapidly, and has no known limitations in assessing liver function compared to other methods, with the exception of detecting hyperbilirubinemia. Unlike existing methods, such as ultrasound or biochemical testing, ICG testing can be conveniently performed at the patient's bedside in realtime.

ICG is a non-toxic, low cost, widely available, and effective fluorescent dye. It emits light in the nearinfrared spectrum, which permits relatively deep tissue penetration. Following intravenous injection, ICG attaches to plasma proteins and allows for easy detection of blood perfusion in real-time. Moreover, ICG is efficiently and selectively taken up by hepatocytes, and can accurately predict liver function preoperatively, intraoperatively, and postoperatively. Preoperatively, ICG detection and clearance rates can provide clinical data on the status of potential surgical candidates, which can help expedite the waiting list. Additionally, ICG can accurately evaluate graft function, so that grafts predicted to be high-risk or ineffective can be avoided. Intraoperatively, surgeons can use ICG to detect anastomosis of the bile duct and blood vessels, which can help prevent biliary leaks and other related complications. Postoperatively, ICG clearance rate can predict early mortality and complications, evaluate graft function and regeneration, and help avoid failures caused by post-transplant complications. The metabolic function of ICG combined with PVF can quantify liver regeneration postoperatively to prevent early complications and help perform appropriate perioperative interventions and management. ICG kinetics evaluate the optimal systemic hemodynamics to increase the success of transplantation.

In short, ICG can be used for various stages of LT, offering unique advantages. We suggest ICG can and should be routinely used in LT to help re-organize the transplant waiting list and improve the surgical outcomes of LT patients.

\section{Acknowledgments}

Funding: None.

\section{Footnote}

Reporting Checklist: The authors have completed the Narrative Review reporting checklist. Available at https:// atm.amegroups.com/article/view/10.21037/atm-21-6650/rc

Conflicts of Interest: All authors have completed the ICMJE uniform disclosure form (available at https://atm. amegroups.com/article/view/10.21037/atm-21-6650/coif). HC is from Nanjing Nuoyuan Medical Devices Co., Ltd. The other authors have no conflicts of interest to declare.

Ethical Statement: The authors are accountable for all aspects of the work in ensuring that questions related to the accuracy or integrity of any part of the work are appropriately investigated and resolved.

Open Access Statement: This is an Open Access article distributed in accordance with the Creative Commons Attribution-NonCommercial-NoDerivs 4.0 International License (CC BY-NC-ND 4.0), which permits the noncommercial replication and distribution of the article with the strict proviso that no changes or edits are made and the original work is properly cited (including links to both the formal publication through the relevant DOI and the license). See: https://creativecommons.org/licenses/by-nc-nd/4.0/.

\section{References}

1. Klein KB, Stafinski TD, Menon D. Predicting survival after liver transplantation based on pre-transplant MELD score: a systematic review of the literature. PLoS One 2013;8:e80661.

2. VanWagner LB, Lapin B, Levitsky J, et al. High early 
cardiovascular mortality after liver transplantation. Liver Transpl 2014;20:1306-16.

3. Tovikkai C, Charman SC, Praseedom RK, et al. Timevarying impact of comorbidities on mortality after liver transplantation: a national cohort study using linked clinical and administrative data. BMJ Open 2015;5:e006971.

4. Piardi T, Lhuaire M, Bruno O, et al. Vascular complications following liver transplantation: A literature review of advances in 2015. World J Hepatol 2016;8:36-57.

5. Rao HB, Prakash A, Sudhindran S, et al. Biliary strictures complicating living donor liver transplantation: Problems, novel insights and solutions. World J Gastroenterol 2018;24:2061-72.

6. Miller CM, Quintini C, Dhawan A, et al. The International Liver Transplantation Society Living Donor Liver Transplant Recipient Guideline. Transplantation 2017;101:938-44.

7. Bedreli S, Sowa JP, Gerken G, et al. Management of acuteon-chronic liver failure: rotational thromboelastometry may reduce substitution of coagulation factors in liver cirrhosis. Gut 2016;65:357-8.

8. Trotter JF, Olson J, Lefkowitz J, et al. Changes in international normalized ratio (INR) and model for endstage liver disease (MELD) based on selection of clinical laboratory. Am J Transplant 2007;7:1624-8.

9. Hoekstra LT, de Graaf W, Nibourg GA, et al. Physiological and biochemical basis of clinical liver function tests: a review. Ann Surg 2013;257:27-36.

10. Jiang JX, Keating JJ, Jesus EM, et al. Optimization of the enhanced permeability and retention effect for nearinfrared imaging of solid tumors with indocyanine green. Am J Nucl Med Mol Imaging 2015;5:390-400.

11. Vos JJ, Wietasch JK, Absalom AR, et al. Green light for liver function monitoring using indocyanine green? An overview of current clinical applications. Anaesthesia 2014;69:1364-76.

12. Struk S, Honart JF, Qassemyar Q, et al. Use of indocyanine green angiography in oncological and reconstructive breast surgery. Ann Chir Plast Esthet 2018;63:54-61.

13. Son GM, Kwon MS, Kim Y, et al. Quantitative analysis of colon perfusion pattern using indocyanine green (ICG) angiography in laparoscopic colorectal surgery. Surg Endosc 2019;33:1640-9.

14. Gilmore DM, Khullar OV, Jaklitsch MT, et al. Identification of metastatic nodal disease in a phase 1 dose-escalation trial of intraoperative sentinel lymph node mapping in non-small cell lung cancer using near-infrared imaging. J Thorac Cardiovasc Surg 2013;146:562-70; discussion 569-70.

15. Herrera-Almario G, Patane M, Sarkaria I, et al. Initial report of near-infrared fluorescence imaging as an intraoperative adjunct for lymph node harvesting during robot-assisted laparoscopic gastrectomy. J Surg Oncol 2016;113:768-70.

16. Kokudo N, Ishizawa T. Clinical application of fluorescence imaging of liver cancer using indocyanine green. Liver Cancer 2012;1:15-21.

17. Haegele S, Reiter S, Wanek D, et al. Perioperative NonInvasive Indocyanine Green-Clearance Testing to Predict Postoperative Outcome after Liver Resection. PLoS One 2016;11:e0165481.

18. Fosby B, Melum E, Bjøro K, et al. Liver transplantation in the Nordic countries - An intention to treat and post-transplant analysis from The Nordic Liver Transplant Registry 1982-2013. Scand J Gastroenterol 2015;50:797-808.

19. Asrani SK, Kamath PS. Model for end-stage liver disease score and MELD exceptions: 15 years later. Hepatol Int 2015;9:346-54.

20. Kamath PS, Kim WR; Advanced Liver Disease Study Group. The model for end-stage liver disease (MELD). Hepatology 2007;45:797-805.

21. Ecochard M, Boillot O, Guillaud O, et al. Could metabolic liver function tests predict mortality on waiting list for liver transplantation? A study on 560 patients. Clin Transplant 2011;25:755-65.

22. Merle U, Sieg O, Stremmel W, et al. Sensitivity and specificity of plasma disappearance rate of indocyanine green as a prognostic indicator in acute liver failure. BMC Gastroenterol 2009;9:91.

23. Routh D, Sharma S, Naidu CS, et al. Comparison of outcomes in ideal donor and extended criteria donor in deceased donor liver transplant: a prospective study. Int J Surg 2014;12:774-7.

24. Nure E, Lirosi MC, Frongillo F, et al. Overextended Criteria Donors: Experience of an Italian Transplantation Center. Transplant Proc 2015;47:2102-5.

25. Jiménez-Castro MB, Gracia-Sancho J, Peralta C. Brain death and marginal grafts in liver transplantation. Cell Death Dis 2015;6:e1777.

26. Tang Y, Han M, Chen M, et al. Donor Indocyanine Green Clearance Test Predicts Graft Quality and Early Graft Prognosis After Liver Transplantation. Dig Dis Sci 2017;62:3212-20. 
27. Zarrinpar A, Lee C, Noguchi E, et al. A rapid, reproducible, noninvasive predictor of liver graft survival. J Surg Res 2015;197:183-90.

28. Wang W, Zhao Q, Hu A, et al. Application of Indocyanine Green (ICG) Detection in Evaluating Early Prognosis in Patients with Fatty Liver Disease After Liver Transplantation. Ann Transplant 2017;22:208-14.

29. Mizuguchi T, Kawamoto M, Meguro M, et al. Preoperative liver function assessments to estimate the prognosis and safety of liver resections. Surg Today 2014;44:1-10.

30. Jeong S, Wang X, Wan P, et al. Risk factors and survival outcomes of biliary complications after adult-to-adult living donor liver transplantation. United European Gastroenterol J 2017;5:997-1006.

31. Kaltenborn A, Gutcke A, Gwiasda J, et al. Biliary complications following liver transplantation: Singlecenter experience over three decades and recent risk factors. World J Hepatol 2017;9:147-54.

32. Nemes B, Gámán G, Doros A. Biliary complications after liver transplantation. Expert Rev Gastroenterol Hepatol 2015;9:447-66.

33. Ishizawa T, Bandai Y, Ijichi M, et al. Fluorescent cholangiography illuminating the biliary tree during laparoscopic cholecystectomy. Br J Surg 2010;97:1369-77.

34. Mitsuhashi N, Kimura F, Shimizu H, et al. Usefulness of intraoperative fluorescence imaging to evaluate local anatomy in hepatobiliary surgery. J Hepatobiliary Pancreat Surg 2008;15:508-14.

35. Mizuno S, Inoue H, Tanemura A, et al. Biliary complications in 108 consecutive recipients with ductto-duct biliary reconstruction in living-donor liver transplantation. Transplant Proc 2014;46:850-5.

36. Mizuno S, Isaji S. Indocyanine green (ICG) fluorescence imaging-guided cholangiography for donor hepatectomy in living donor liver transplantation. Am J Transplant 2010;10:2725-6.

37. Hong SK, Suh KS, Kim HS, et al. Pediatric Living Donor Liver Transplantation Using a Monosegment Procured by Pure 3D Laparoscopic Left Lateral Sectionectomy and In situ Reduction. J Gastrointest Surg 2018;22:1135-6.

38. Khalaf H. Vascular complications after deceased and living donor liver transplantation: a single-center experience. Transplant Proc 2010;42:865-70.

39. Pawlak J, Grodzicki M, Leowska E, et al. Vascular complications after liver transplantation. Transplant Proc 2003;35:2313-5.

40. Duffy JP, Hong JC, Farmer DG, et al. Vascular complications of orthotopic liver transplantation: experience in more than 4,200 patients. J Am Coll Surg 2009;208:896-903; discussion 903-5.

41. Mori K, Nagata I, Yamagata S, et al. The introduction of microvascular surgery to hepatic artery reconstruction in living-donor liver transplantation--its surgical advantages compared with conventional procedures. Transplantation 1992;54:263-8.

42. Kubota K, Kita J, Shimoda M, et al. Intraoperative assessment of reconstructed vessels in living-donor liver transplantation, using a novel fluorescence imaging technique. J Hepatobiliary Pancreat Surg 2006;13:100-4.

43. Azoulay D, Linhares MM, Huguet E, et al. Decision for retransplantation of the liver: an experience- and costbased analysis. Ann Surg 2002;236:713-21; discussion 721.

44. Olthoff KM, Kulik L, Samstein B, et al. Validation of a current definition of early allograft dysfunction in liver transplant recipients and analysis of risk factors. Liver Transpl 2010;16:943-9.

45. Figueroa R, Golse N, Alvarez FA, et al. Indocyanine green fluorescence imaging to evaluate graft perfusion during liver transplantation. HPB (Oxford) 2019;21:387-92.

46. Kawaguchi Y, Akamatsu N, Ishizawa T, et al. Evaluation of hepatic perfusion in the liver graft using fluorescence imaging with indocyanine green. Int J Surg Case Rep 2015;14:149-51.

47. Akamatsu N, Sugawara Y, Kaneko J, et al. Effects of middle hepatic vein reconstruction on right liver graft regeneration. Transplantation 2003;76:832-7.

48. Lee S, Park K, Hwang S, et al. Congestion of right liver graft in living donor liver transplantation. Transplantation 2001;71:812-4.

49. Sano K, Makuuchi M, Miki K, et al. Evaluation of hepatic venous congestion: proposed indication criteria for hepatic vein reconstruction. Ann Surg 2002;236:241-7.

50. Hashimoto T, Miki K, Imamura H, et al. Sinusoidal perfusion in the veno-occlusive region of living liver donors evaluated by indocyanine green and near-infrared spectroscopy. Liver Transpl 2008;14:872-80.

51. Kawaguchi Y, Ishizawa T, Miyata Y, et al. Portal uptake function in veno-occlusive regions evaluated by real-time fluorescent imaging using indocyanine green. J Hepatol 2013;58:247-53.

52. Ogura Y, Hori T, El Moghazy WM, et al. Portal pressure $<15 \mathrm{~mm} \mathrm{Hg}$ is a key for successful adult living donor liver transplantation utilizing smaller grafts than before. Liver Transpl 2010;16:718-28.

53. Yagi S, Iida T, Taniguchi K, et al. Impact of portal venous pressure on regeneration and graft damage after living- 


\section{Page 10 of 10}

donor liver transplantation. Liver Transpl 2005;11:68-75.

54. Hori T, Ogura Y, Yagi S, et al. How do transplant surgeons accomplish optimal portal venous flow during livingdonor liver transplantation? Noninvasive measurement of indocyanine green elimination rate. Surg Innov 2014;21:43-51.

55. Kim YS, Lim HK, Rhim H, et al. Recurrence of hepatocellular carcinoma after liver transplantation: patterns and prognostic factors based on clinical and radiologic features. AJR Am J Roentgenol 2007;189:352-8.

56. Valdivieso A, Bustamante J, Gastaca M, et al. Management of hepatocellular carcinoma recurrence after liver transplantation. Transplant Proc 2010;42:660-2.

57. Ikegami T, Hashikura Y, Nakazawa Y, et al. Risk factors contributing to hepatic artery thrombosis following livingdonor liver transplantation. J Hepatobiliary Pancreat Surg 2006;13:105-9.

58. Du Z, Wei Y, Chen K, et al. Risk factors and criteria predicting early graft loss after adult-to-adult living donor liver transplantation. J Surg Res 2014;187:673-82.

59. Olmedilla L, Pérez-Peña JM, Ripoll C, et al. Early noninvasive measurement of the indocyanine green plasma disappearance rate accurately predicts early graft dysfunction and mortality after deceased donor liver transplantation. Liver Transpl 2009;15:1247-53.

60. Klinzing S, Brandi G, Stehberger PA, et al. The combination of MELD score and ICG liver testing predicts length of stay in the ICU and hospital mortality in liver transplant recipients. BMC Anesthesiol 2014;14:103.

Cite this article as: Dai B, Guissi NEI, Sulyok LF, Bryski MG, Wang Y, Wang D, Singhal S, Cai H. Advantages of using indocyanine green in liver transplantation: a narrative review. Ann Transl Med 2022;10(2):110. doi: 10.21037/atm-21-6650

\section{Dai et al. Advantages of indocyanine green in liver transplantation}

61. Levesque E, Saliba F, Benhamida S, et al. Plasma disappearance rate of indocyanine green: a tool to evaluate early graft outcome after liver transplantation. Liver Transpl 2009;15:1358-64.

62. Levesque E, Hoti E, Azoulay D, et al. Non-invasive ICGclearance: a useful tool for the management of hepatic artery thrombosis following liver transplantation. Clin Transplant 2011;25:297-301.

63. Sun Y, Yu L, Liu Y. Predictive Value of Indocyanine Green Plasma Disappearance Rate on Liver Function and Complications After Liver Transplantation. Med Sci Monit 2018;24:3661-9.

64. Vos JJ, Scheeren TW, Lukes DJ, et al. Intraoperative ICG plasma disappearance rate helps to predict absence of early postoperative complications after orthotopic liver transplantation. J Clin Monit Comput 2013;27:591-8.

65. Hori T, Iida T, Yagi S, et al. K(ICG) value, a reliable real-time estimator of graft function, accurately predicts outcomes in adult living-donor liver transplantation. Liver Transpl 2006;12:605-13.

66. Jochum C, Beste M, Penndorf V, et al. Quantitative liver function tests in donors and recipients of living donor liver transplantation. Liver Transpl 2006;12:544-9.

67. Eguchi S, Yanaga K, Sugiyama N, et al. Relationship between portal venous flow and liver regeneration in patients after living donor right-lobe liver transplantation. Liver Transpl 2003;9:547-51.

(English Language Editor: A. Kassem) 